Revue musicale OICRM

\title{
Le cas Constantinople. Historique, identité musicale et enjeux de la professionnalisation des musiciens migrants
}

\section{Caroline Marcoux-Gendron}

Volume 1, numéro 1, 2012

Profession musicale au Québec

URI : https://id.erudit.org/iderudit/1055857ar

DOI : https://doi.org/10.7202/1055857ar

Aller au sommaire du numéro

Éditeur(s)

OICRM

ISSN

2368-7061 (numérique)

Découvrir la revue

Citer cet article

Marcoux-Gendron, C. (2012). Le cas Constantinople. Historique, identité musicale et enjeux de la professionnalisation des musiciens migrants. Revue musicale OICRM, 1(1), 36-56. https://doi.org/10.7202/1055857ar
Résumé de l'article

La notion de professionnalisation appliquée aux musiciens migrants pose plusieurs défis en sociomusicologie, car elle combine l'impossibilité d'établir un profil de carrière valable pour tous en musique à la nécessité de prendre en compte plusieurs dimensions autres que strictement socioprofessionnelles pour bien appréhender le processus d'insertion de l'artiste dans son nouvel environnement. C'est à Montréal que cette étude fut menée auprès de la formation Constantinople qui fait appel à plusieurs musiciens nés à l'extérieur du pays. L'article visera d'abord à retracer les trajectoires individuelles des musiciens migrants Didem Basar, Liu Fang, Kiya Tabassian et Ziya Tabassian depuis leur arrivée dans la métropole. Puis, il sera question de

professionnalisation à travers la reconstitution de l'histoire de Constantinople. Ceci permettra de comprendre comment l'ensemble s'est structuré et affirmé au sein de la vie musicale québécoise, et comment il a agi comme espace de recherche d'une identité musicale pour ses membres. 


\title{
Le cas Constantinople. Historique, identité musicale et enjeux de la professionnalisation des musiciens migrants
}

\author{
Caroline Marcoux-Gendron
}

\begin{abstract}
Résumé
La notion de professionnalisation appliquée aux musiciens migrants pose plusieurs défis en sociomusicologie, car elle combine l'impossibilité d'établir un profil de carrière valable pour tous en musique à la nécessité de prendre en compte plusieurs dimensions autres que strictement socioprofessionnelles pour bien appréhender le processus d'insertion del'artiste dans son nouvel environnement. C'està Montréal que cette étude fut menée auprès de la formation Constantinople qui fait appel à plusieurs musiciens nés à l'extérieur du pays. L'article visera d'abord à retracer les trajectoires individuelles des musiciens migrants Didem Basar, Liu Fang, Kiya Tabassian et Ziya Tabassian depuis leur arrivée dans la métropole. Puis, il sera question de professionnalisation à travers la reconstitution de l'histoire de Constantinople. Ceci permettra de comprendre comment l'ensemble s'est structuré et affirmé au sein de la vie musicale québécoise, et comment il a agi comme espace de recherche d'une identité musicale pour ses membres.
\end{abstract}

Mots clés: ensemble Constantinople ; identité musicale ; insertion socioprofessionnelle ; milieu musical montréalais ; musiciens migrants.

\footnotetext{
Abstract

The concept of professionalization applied to migrant musicians raises a number of challenges in sociomusicology, because it combines the impossibility to establish a career profile valid for all in music and the need to take into account several dimensions other than strictly socioprofessional to understand the process of the artist's integration in his new environment. This study was conducted in Montreal with the Constantinople Ensemble which involves several musicians born outside the country. First, the article aims to trace the individual trajectories of the migrant musicians Didem Basar, Liu Fang, Kiya Tabassian and Ziya Tabassian since their arrival in the city. Then, we will discuss the professionalization through the reconstruction of Constantinople history. This will help to understand how the ensemble has been structured, how it has established itself in Quebec musical life, and how it has acted for its members as a space for the search for a musical identity.

Keywords: Constantinople Ensemble; migrant musicians; Montreal's music scene; musical identity; socioprofessional integration.
} 
La notion de professionnalisation pose plusieurs défis en sociomusicologie ${ }^{1}$ dans la mesure où il s'avère impossible d'établir un profil de carrière ${ }^{2}$ qui soit valable pour tous les musiciens. Mener une enquête à ce sujet auprès d'artistes migrants ajoute aux considérations socioprofessionnelles des enjeux d'autres ordres, car ces individus ont à se positionner comme musiciens à part entière tout en affirmant un bagage de pratiques et de connaissances musicales qui leur est propre. À ce propos, Montréal constitue un lieu de choix pour une étude, s'agissant d'une métropole dont la composition sociodémographique est très diversifiée ${ }^{3}$, et qui accueille ainsi de nombreux musiciens venus d'ailleurs, aux profils et aux parcours variés.

Cette enquête de terrain s'est arrêtée sur un groupe déjà bien établi dans le paysage musical québécois : la formation Constantinople. Articulée autour des frères Tabassian, deux musiciens d'origine iranienne établis au Québec depuis leur adolescence, Constantinople se veut un lieu de rencontres entre plusieurs répertoires musicaux, de la musique ancienne à la musique de " traditions vivantes ${ }^{4}$ ", provenant tant de l'Europe méditerranéenne, du Moyen-Orient que du Nouveau Monde. Rencontres de traditions à travers la musique donc, mais également à travers les musiciens, car Constantinople a vu défiler un grand nombre d'artistes depuis ses débuts, qu'ils aient été des membres fixes ou des collaborateurs sporadiques. De plus, le choix de cet ensemble apparaissait particulièrement pertinent pour la question de l'insertion professionnelle, car si plusieurs de ses membres ont reçu leur formation musicale au Canada, d'autres ont été formés dans leur pays d'origine avant d'immigrer au Québec 5 .

L'enquête s'est échelonnée sur une période de neuf mois, de juin 2011 à février 2012, et a permis de rencontrer six musiciens pour des entretiens semi-dirigés

$1 \quad$ Plusieurs auteurs abordent la notion de professionnalisation en musique à travers des études qui touchent différentes réalités contemporaines. À titre d'exemple, Marc Perrenoud s'intéresse à la réalité sociale des instrumentistes dits « ordinaires » dans Les musicos (2007) ; Morgan Jouvenet (2006) se penche sur la professionnalisation en musique rap ; Hyacinthe Ravet (2006), sur celle des femmes dans les orchestres de musique classique occidentale, etc.

2 En art, la notion de « carrière » se détache de son sens strict, à savoir un parcours standardisé fait d'un enchaînement de positions hiérarchiquement ordonnées. La trajectoire d'un artiste adopte davantage une forme de «ligne brisée » (Sibaud 2013, p. 184), faite d'une succession de positions sans ordre formalisé. Pour Nathalie Heinich, réduire la carrière d'un artiste à un plan ou à une stratégie est précisément contraire au domaine artistique (Heinich 1998, p. 19) ; il s'agit plutôt d'un cheminement professionnel qui repose essentiellement, dans les arts du spectacle comme la musique, sur une dynamique par projets (Menger 2009, p. 565-566).

3 Montréal est souvent caractérisée par sa " diversité urbaine », 86,9\% de la population immigrée du Québec se concentrant dans cette région métropolitaine de recensement (RMR) (MICC 2009, p. 20). Aussi, des statistiques sur la population active dans les arts au Canada au tournant des années 2000 révélaient qu'il s'y trouvait 3000 artistes immigrants, soit 16\% de tous les artistes de Montréal (Hill Strategies 2005, p. 28).

4 L'expression «traditions vivantes » est notamment utilisée par l'Unesco dans le cadre de son action pour la sauvegarde du patrimoine culturel immatériel. Sont considérées comme expressions ou traditions vivantes les «traditions orales, les arts du spectacle, les pratiques sociales, rituels et évènements festifs, les connaissances et pratiques concernant la nature et l'univers ou les connaissances et le savoir-faire nécessaires à l'artisanat traditionnel » (Unesco 2015). En somme, même si ces traditions découlent souvent d'une transmission ancestrale, elles font l'objet d'une pratique actualisée, d'où l'usage du qualificatif « vivant ».

5 Les frères Tabassian, ainsi que les deux musiciens d'origine canadienne Matthew Jennejohn et Pierre-Yves Martel, ont étudié au Canada, tandis que Liu Fang a été formée en Chine et Didem Basar en Turquie. 
de plus ou moins une heure ${ }^{6}$. Les participants à cette étude sont Kiya Tabassian, fondateur et directeur artistique de Constantinople ; Ziya Tabassian, percussionniste et membre depuis les débuts ; Didem Basar, musicienne d'origine turque et collaboratrice fréquente depuis quelques années ; Liu Fang, instrumentiste d'origine chinoise ayant joué avec le groupe en 2000 ; Matthew Jennejohn, musicien d'origine canadienne et membre fixe pendant les quatre premières saisons de la formation ; et Pierre-Yves Martel, artiste d'origine canadienne et l'un des principaux membres actuels. À cette enquête de terrain s'ajoute une émission de radio diffusée sur les ondes de la Première Chaîne de Radio-Canada en décembre 2010 : "Nous sommes des vôtres ", animée par Élizabeth Gagnon. Les frères Tabassian y parlaient de leur arrivée au Québec et de leur cheminement artistique en passant par les étapes de leur carrière actuelle. Finalement, quelques recherches biographiques et discographiques sur les musiciens ont complété ces sources.

Cet article vise à retracer le processus de professionnalisation des musiciens migrants depuis leur arrivée à Montréal, problématique qui sera explorée sous deux angles.

D'une part, il sera question des trajectoires individuelles des quatre musiciens migrants Didem Basar, Liu Fang, Kiya Tabassian et Ziya Tabassian. Leur cheminement professionnel dans le milieu musical montréalais sera examiné à travers des considérations de plusieurs ordres : culturel, linguistique, social. D'autre part, il s'agira plus spécifiquement de la société musicale Constantinople ${ }^{7}$ et de sa structuration à travers les années, afin de comprendre comment elle s'est intégrée et affirmée dans la vie culturelle québécoise, mais aussi comment elle a agi comme espace d'expérimentations et de recherche musicales pour ses membres. Cette partie de l'article reposera sur une analyse diachronique, soit la reconstitution de l'histoire de la formation. C'est à travers les témoignages des participants de l'enquête et grâce à des données tirées des sites du Conseil des arts de Montréal (CAM), du Conseil des arts et des lettres du Québec (CALQ) et du Conseil des arts du Canada (CAC) que cet historique a pu être établi.

\section{TRAJECTOIRES DE MUSICIENS MigRANTS À MONTRÉAL}

Les musiciens Didem Basar, Liu Fang, Kiya Tabassian et Ziya Tabassian partagent une expérience de vie commune : celle d'avoir immigré au Québec. Or, les circonstances entourant leur venue diffèrent beaucoup. Kiya et Ziya Tabassian, arrivés en 1990 respectivement à l'âge de 14 ans et de 11 ans, ont immigré avec leurs parents, tandis que Didem Basar, au Québec depuis 2007, s'est installée à Montréal au début de la trentaine, et que Liu Fang est arrivée en 1996, au début de la vingtaine. Ces deux

6 Cette recherche impliquant des êtres humains a reçu l'aval du Comité plurifacultaire d'éthique de la recherche de l'Université de Montréal dont relève la Faculté de musique.

7 L'expression " société musicale » sera récurrente dans cet article et réfère à l'idée d'un petit groupe de musiciens réunis autour d'intérêts communs, interagissant selon des « règles » et des codes précis au sein d'une organisation où s'observe une certaine hiérarchie et où s'opèrent des prises de décisions. Il s'agit donc d'envisager Constantinople comme une « société » au niveau microscopique. 
femmes ont choisi d'immigrer d'abord et avant tout pour accompagner leur mari qui rencontrait de nouvelles opportunités professionnelles.

$\mathrm{Du}$ point de vue musical, plusieurs caractéristiques unissent ces musiciens. D'une part, ils ont tous appris une tradition musicale orale, mais dont la pratique s'est institutionnalisée et, jusqu'à un certain point, a connu une forme de fixation sur papier. D'autre part, ces instrumentistes possédaient déjà un statut de musicien dans leur pays d'origine, à divers degrés considérant l'âge de leur départ pour le Québec. Dans le cas des frères Tabassian, bien que très jeunes, ils avaient déjà formé un petit groupe de musique avec des camarades d'école et avaient gagné des concours, joué en spectacles et passé sur les ondes de la radio et à la télévision nationale d'Iran. Quant à Liu Fang, joueuse de pipa et de guzheng, ses premières apparitions en concert remontent à son enfance. Aussi, dès sa sortie du Conservatoire, elle a entamé une carrière qui ne dura guère puisqu'elle a quitté la Chine très vite. Finalement, Didem Basar faisait partie de plusieurs groupes musicaux actifs en Turquie et à l'étranger, formations au sein desquelles elle jouait le qanun et, occasionnellement, le santur turc.

Enfin, un autre point de convergence de leurs profils est que chacun maîtrise plusieurs traditions musicales distinctes, ce que d'aucuns qualifient de «bi-musicalité » suivant les travaux de l'ethnomusicologue Mantle $\mathrm{Hood}^{8}$. Pour les musiciens de cette étude, il s'agit de la tradition musicale occidentale et de la tradition liée à leur pays d'origine.

Or, quelques similitudes n'impliquent pas des parcours en tous points semblables. L'expérience migratoire de ces " passeurs de musique ", comme les appelle l'ethnomusicologue Laurent Aubert, recouvre plusieurs dimensions : "Les musiciens migrants sont [...] amenés à se situer par rapport à leur culture d'origine, tout comme ils doivent trouver leur place dans leur nouvel environnement, y compris vis-à-vis de leur propre diaspora » (Aubert 2005, p. 116). Cette première partie de l'article se structure autour de cette affirmation en ce qu'il s'agira de retracer la trajectoire de chaque musicien en interrogeant son rapport à la culture de son pays d'origine - qui sera ici limitée à la musique -, puis à son nouvel environnement, d'une part en regard des membres de sa propre " diaspora ${ }^{9}$ ", d'autre part vis-à-vis du milieu montréalais dans son ensemble.

8 Le terme «bi-musicalité » a été critiqué par Mantle Hood lui-même, qui en explique les raisons dans son entrevue avec Giuriati. L'ethnomusicologue affirme néanmoins que le principe à la base de ce terme a aujourd'hui été accepté dans le monde entier (Giuriati et Hood 1995, p. 202-203).

9 De par ses nombreuses occurrences dans la littérature, le terme "diaspora " a vu son champ sémantique s'élargir et recoupe désormais plusieurs autres termes relatifs aux phénomènes migratoires. Pour certains auteurs, la " diaspora " doit répondre à plusieurs critères (historique de dispersion des membres, maintien d'une mémoire autour du pays d'origine et projet de retour, etc.), mais la perspective d'un « idéal-type » trop rigide est également débattue (Clifford 1994, p. 303-307). En somme, le terme fait l'objet de plusieurs réserves à l'heure actuelle, et il ne sera conservé dans cet article que pour faire écho à la citation de Laurent Aubert. 


\section{Le rapport à la tradition musicale du pays d'origine}

Le lien de chacun à la tradition musicale de son pays d'origine transparaît notamment à travers le discours tenu sur la musique. D'emblée, les quatre musiciens ont témoigné d'un grand respect pour ces traditions musicales ancestrales que sont les traditions chinoise, persane et turque. Chacun a consacré beaucoup de temps à l'apprentissage et au perfectionnement de ces pratiques. Or, ils ont aussi admis, à différents moments de leurs parcours et à différents degrés, un besoin à la fois personnel et créatif de "transcender » les codes de ces traditions, de sortir du cadre strict qui les balise afin de procéder à une réappropriation, à une réinterprétation plus personnelle de ces musiques ${ }^{10}$.

Pour Kiya Tabassian dont les actuels projets au sein de Constantinople reflètent bien cette envie d'exploration, un besoin de « rester connecté » a par ailleurs caractérisé les premiers moments passés au Québec, ce que le musicien a fait en fixant toute son attention sur son instrument, le sétar.

\section{Exemple audio 1 : Kiya Tabassian en entrevue. Écouter.}

Selon ce musicien, l'enregistrement de l'album Jardin de la mémoire (2000) avec son frère Ziya, alors qu'ils étaient devenus de jeunes adultes, aurait marqué une rupture dans leur attitude plus stricte envers la tradition musicale persane. Après cet enregistrement, Kiya parle d'une nouvelle étape visant désormais à " trouver une couleur d'[eux]-mêmes » (Kiya Tabassian, décembre 2011) ${ }^{11}$. Cet épisode marque pour lui le passage d'une attitude plus conservatrice à une démarche résolument personnelle, qui puise certes dans la tradition, mais cherche également à en dépasser les prescriptions.

Didem Basar a exprimé un désir semblable, elle qui a d'ailleurs sciemment changé de département à l'Université technique d'Istanbul pour étudier la composition après avoir complété une formation en instrument (le qanun). Dans son deuxième cursus, elle a pu suivre des cours de théorie musicale occidentale. Même si cet apprentissage a d'abord été difficile de son propre aveu, il lui a permis d'élargir sa vision du monde musical et d'appliquer des principes harmoniques, polyphoniques au qanun, un instrument qui repose sur une tradition musicale monodique. Depuis son arrivée à Montréal, Didem Basar poursuit ses explorations musicales en effectuant des collaborations avec des musiciens de tous horizons (en jazz et musique tzigane notamment), ce qui contribue à transformer son regard sur la musique et à faire émerger de nouvelles

10 Dans l'article «Les passeurs de musique », Aubert discute du rapport de certains artistes emblématiques (ex. : Ravi Shankar et Munir Bashir) à la musique traditionnelle qu'ils pratiquent et constate chez chacun à la fois une formation, une connaissance très développée de cette musique, mais aussi un style résolument personnel, une sorte de rénovation du langage musical. L'auteur rappelle aussi le caractère vivant de toute tradition, qui évolue et se développe avec le temps, la « question [étant] plutôt de savoir où se situe le point de rupture à partir duquel une expression individuelle cesse de répondre aux critères de la tradition dont elle émane» (Aubert 2005, p. 126).

11 De telles références renvoient aux entretiens effectués pendant le terrain d'enquête. Les courts extraits seront transcrits à même le texte, alors que les longues citations se présenteront sous format audio pour ne pas surcharger l'article. 
idées créatrices admet-elle. Ainsi, lorsqu'il est question de sa manière d'interpréter la musique turque aujourd'hui, elle répond ne pas le faire de façon très conventionnelle, car " c'est important de rompre le cadre " (Didem Basar, février 2012) ${ }^{12}$.

Quant à Liu Fang, elle s'adonne également à toutes sortes de collaborations, dans des répertoires dits traditionnels comme contemporains, et parle de l'intérêt des échanges entre musiciens d'horizons différents ${ }^{13}$. Ses propos relatifs à la tradition musicale chinoise sont davantage empreints de l'idée d' " authenticité ", de fidélité à cet art, ce qui passe par ailleurs par un long travail d'appropriation des œuvres, par la nécessité de mettre de soi dans chaque interprétation, explique-t-elle. Contrairement aux autres musiciens cependant, elle ne compose pas ; si elle dit y penser souvent, elle admet ne pas se sentir prête devant la forme de grandeur que revêt pour elle la musique qu'elle pratique.

Ainsi, le rapport de chaque musicien à la tradition musicale de son pays d'origine se transforme au fil du temps. De plus, ce rapport s'exprime différemment à travers le discours et se vit de manière distincte en considérant la relation de chacun à l'interprétation et à la composition musicales.

\section{Le rapport à la " diaspora»}

En ce qui concerne le rapport du musicien aux membres de sa « diaspora » également installés à Montréal, les expériences individuelles présentent aussi des nuances. Le sujet sera exploré à la fois en regard des pairs musiciens et du public montréalais de même origine que l'artiste.

Tout d'abord, le parcours des frères Tabassian illustre bien la transformation de la relation d'un individu migrant au groupe de même origine dans le pays d'installation, à savoir la " communauté culturelle des Iraniens " comme l'a nommée l'aîné Kiya $^{14}$. Après quelques mois passés au Québec, ce dernier a fait la rencontre d'un musicien d'origine iranienne qui l'a invité à se produire lors d'un spectacle pour la communauté iranienne de Montréal. Puis, la rencontre d'un autre musicien de même origine a abouti à une solide amitié de laquelle est né un projet de trio, le Darvish Khan, dont Ziya était d'ailleurs le troisième membre. Cette formation, qui explorait la tradition musicale persane, recevait du financement de la communauté iranienne pour se produire à Montréal et à Toronto. Puis, Kiya a petit à petit senti le besoin de sortir du réseau communautaire, et c'est par les maisons de la culture que cet affranchissement a pu avoir lieu, affirme-t-i1 ${ }^{15}$.

12 Traduction personnelle de la phrase : "It is important to break the frame ».

13 Liu Fang a affirmé en entrevue qu'il est important d'aller " out of the circle of pipa, to learn from other musicians: guitar players, cellos, to learn from piano, to learn from other musicians, to see what they do » (Liu Fang, novembre 2011).

14 La référence à une " communauté » se limitera aux situations où le terme a été employé par le musicien même en entretien. La " communauté " sera ainsi appréhendée à travers l'expérience, les perceptions des individus ; elle sera envisagée telle une construction subjective, symbolique (Chevalier 2007).

15 Si les maisons de la culture sont ici présentées comme un lieu d'émancipation, d'affranchissement du réseau communautaire iranien, c'est parce que les concerts n'y étaient alors plus financés par ladite 
Aujourd'hui, les frères Tabassian multiplient les collaborations avec des musiciens de toutes origines et disent compter sur un public très diversifié avec Constantinople, public qui ne se compose pas forcément d'individus d'origine iranienne, sauf peut-être lors de leurs concerts spécialement dédiés à la tradition musicale persane, avance Kiya en entretien ${ }^{16}$.

Pour Didem Basar, la toute première rencontre "musicale » à Montréal a été celle d'Ismail Fencioglu, musicien d'origine turque établi dans la métropole depuis le début des années 2000 et dont les contacts communs ont favorisé les premiers échanges Fencioglu a étudié avec la sœur de Basar en Turquie. C'est davantage par lui que la musicienne semble avoir enraciné sa carrière au Québec ${ }^{17}$, alors qu'il l'a introduite à plusieurs artistes et formations musicales, en plus de collaborer directement avec elle.

En bref, Basar participe à plusieurs projets, certains impliquant des musiciens et/ou des répertoires d'origine turque, d'autres non. Elle joue, par exemple, au sein du Duo Turco, formé avec Fencioglu et mêlant tradition musicale turque et compositions originales, de Minor Empire, groupe basé à Toronto qui marie influences traditionnelles turques et sonorités plus contemporaines, tout comme elle accompagne l'accordéoniste d'origine moldave Sergiu Popa, découvre le jazz aux côtés du contrebassiste d'origine canadienne Sage Reynolds, collabore bien sûr avec Constantinople de manière sporadique, etc. Ainsi, Basar a rapidement créé des contacts avec des musiciens aux profils (culturel, musical) très variés. Concernant le public présent à ses concerts, la question a été brièvement touchée lors de l'entretien et la musicienne a estimé qu'il y avait à la fois des individus d'origine turque, mais aussi une portion significative de gens de toutes origines.

Quant à Liu Fang, elle affirme avoir été rapidement amenée à développer de nombreuses collaborations avec des musiciens de toutes origines, en atteste sa feuille de route $^{18}$. Du côté du public, elle déclare que la " communauté asiatique » est minoritaire dans ses concerts montréalais. En somme, l'entretien n'a pas révélé de point d'ancrage marqué pour la musicienne à Montréal, qu'il s'agisse d'un acteur phare (comme Fencioglu pour Basar) ou d'une dite diaspora. Or, le mode de vie de Liu Fang et son mari pourrait alimenter cette situation, eux qui partagent leur temps entre deux continents car les engagements de la musicienne sont plus nombreux en Europe qu'au Canada.

communauté. Les maisons de la culture appartiennent plutôt à un réseau de diffuseurs municipaux (voir Accès culture Montréal 2015) et Kiya Tabassian explique qu'un concert diffusé en ces lieux était acheté par la maison en question.

16 Quelques exemples de concerts spécialement dédiés à ce répertoire : Cri persan en mars 2007, Chemins au sommet en mars 2011, Éclats en novembre 2012.

17 Lors de l'entretien, la musicienne n'a jamais fait référence à une « communauté turque », affirmant seulement qu'il y avait davantage de personnes d'origine turque à Toronto qu'à Montréal. En effet, au Canada, 57\% de la population d'origine turque se trouvent en Ontario, contre 26\% au Québec (Shaker 2013).

18 Voici une liste partielle, mais tout de même révélatrice des collaborations que Liu Fang a faites à ce jour : Nouvel Ensemble Moderne (NEM), Quatuor Alcan, Quatuor Paul Klee, sMCQ, flûtistes Lise Daoust et Henri Tournier, guitariste Michael O'Toole, joueur de kora Ballaké Sissoko, joueur de shakuhachi japonais Yoshio Kurahashi, joueur de tabla Subhashish Bhattacharya, oudiste Farhan Sabbagh, violoncelliste Yegor Dyachkov, violoniste Malcolm Goldstein, etc. 
Bref, la relation de chaque musicien au groupe de même origine installé à Montréal s'avère variable. Par exemple, la référence à une "communauté » n'a pas été systématique, montrant que les relations établies par les individus migrants dans leur nouvel environnement répondent à différents schémas :

Toute approche qui tenterait d'interpréter de façon linéaire appartenance ethnique et modalités d'insertion ferait fausse route ; la "communauté » ethnique ne préexiste pas l'immigration [sic], les relations dont il s'agit sont en grande partie construites à travers l'expérience migratoire (Fortin 2000, p. 24).

\section{Le rapport au nouvel environnement : réseaux et compétences linguistiques}

Quant au rapport des musiciens à leur nouvel environnement de vie et de travail, Montréal, les notions de réseaux et de compétences linguistiques seront privilégiées.

D'abord, la question des réseaux, bien que déjà touchée dans la section traitant de la "diaspora ", sera désormais envisagée de manière plus générale par rapport aux carrières actuelles des musiciens. En sociologie de l'art, cette notion parcourt la littérature : par exemple, Pierre-Michel Menger indique que " chaque artiste appartient à un ou plusieurs réseaux de pairs qui constituent son groupe immédiat de référence, d'évaluation et de soutien » (Menger 2009, p. 564). Dans le milieu musical, les réseaux s'avèrent de fait essentiels, alors que les engagements se contractent souvent par bouche-à-oreille. Non seulement le réseau contribue-t-il à ce que l'artiste soit pressenti pour être engagé, mais il permet aussi à ce dernier d'aller au-devant des choses en obtenant de l'information sur de nouveaux projets auxquels il pourra participer d'une manière ou d'une autre.

À ce titre, les carrières des frères Tabassian offrent de bons exemples de réseautage. Leurs études au Québec ${ }^{19}$ ont certes joué en leur faveur, car l'école représente, dans les domaines artistiques, un lieu privilégié pour de premières rencontres, et éventuellement, de premiers engagements ${ }^{20}$. Tel que 1'affirme le sociologue Pascal NicolasLe Strat, le passage par une école " aide à se socialiser professionnellement [...], à se repérer parmi les multiples devenir-réseaux qui structurent les mondes de l'art » (Nicolas-Le Strat 1998, p. 106). Kiya Tabassian semble particulièrement conscient de l'importance des réseaux ; son discours témoigne de sa lucidité à ce sujet.

Exemple audio 2 : Kiya Tabassian en entrevue. Écouter.

19 Kiya Tabassian a fréquenté l'école secondaire Saint-Luc, l'École de musique Vincent-d'Indy et le Conservatoire de musique de Montréal, alors que Ziya Tabassian est allé à Saint-Luc également pour le secondaire, puis au Cégep Marie-Victorin et à l'Université de Montréal.

20 Françoise Liot développe longuement cette idée selon laquelle l'école favorise l'insertion dans les réseaux institutionnel et professionnel (Liot 2004, p. 184-200). Et si cette auteure traite la question précisément en regard du domaine des beaux-arts, son constat peut s'élargir à un grand nombre d'autres milieux artistiques, y compris celui de la musique. Les frères Tabassian en fournissent un exemple probant et le seul fait que Constantinople soit né de rencontres au Conservatoire de musique de Montréal est déjà significatif à cet égard. 
Le rapport des frères Tabassian aux institutions et organismes locaux, qui se manifeste entre autres par leur engagement au sein de conseils administratifs - au Conseil des arts de Montréal pour Kiya, chez Code d'Accès pour Ziya -, est également important à souligner. En effet, il s'agit d'indices de leur implication, de leur position dans le milieu musical montréalais. À ce propos, le musicien Pierre-Yves Martel, troisième membre régulier de Constantinople ces dernières années, témoigne d'ailleurs : " Je me promène dans la rue avec Kiya, ou Ziya, tout le monde les connaît, ils nous arrêtent tout le temps dans la rue : "Hé salut ! Comment ça va ?". [...] Ils sont vraiment impliqués dans la communauté musicale tu sais... " (Pierre-Yves Martel, juin 2011). Chez les frères Tabassian, la reconnaissance acquise au fil des ans n'est ainsi pas étrangère aux réseaux qu'ils ont développés, à leurs contacts tant interpersonnels qu'institutionnels, ce qui contribue à leur haut degré d'activité musicale dans le milieu professionnel aujourd'hui.

De son côté, Didem Basar admet le rôle indispensable de Fencioglu dans sa carrière au Québec ; ce soutien l'a rendue confiante en l'avenir dans la mesure où elle est de plus en plus sollicitée pour des engagements, et ce, indépendamment du cercle des contacts de Fencioglu. Ce dernier a néanmoins agi comme une sorte de pivot ; il incarne l'une de ces « rencontres » décisives qui ponctuent souvent les récits de vie de musiciens, qui influencent les trajectoires en favorisant une mobilité au sens social, statutaire (Le Menestrel 2012, p. 237-243).

Pour Liu Fang, la question des réseaux a été traitée directement avec son mari, Risheng Wang, car il est également son gérant. Ce dernier signale que la plupart des contrats obtenus résultent de demandes reçues par Internet, admettant du même souffle qu'il n'a pas vraiment le temps ni les outils pour faire du réseautage ${ }^{21}$. À la fin de l'entretien, Risheng Wang a d'ailleurs confié que le couple se sentait encore en marge du milieu musical québécois malgré toutes les années passées ici ${ }^{22}$. En ce sens, la fréquence des concerts de Liu Fang s'avère variable au fil des ans, ce qui est notamment imputable à la fluctuation de l'appui financier reçu par différentes institutions subventionnaires, explique le couple ${ }^{23}$.

En somme, le réseautage est une dimension essentielle dans la carrière professionnelle des musiciens. Dans le cas d'artistes migrants, les réseaux contribuent à l'insertion socioprofessionnelle, certes, mais peuvent aussi être envisagés comme vecteurs d'insertion sociale dans un sens plus large. Ils agissent effectivement comme

21 Risheng Wang est scientifique de formation et c'est pour un emploi dans ce domaine que le couple a immigré au Canada. L'homme a cependant décidé d'abandonner sa carrière en science pour se dédier à la gestion de la carrière de sa femme il y a plusieurs années. Bref, si le couple a d'abord immigré pour le travail du mari, c'est finalement la carrière artistique de Liu Fang qui est devenue leur principale source de revenus familiaux.

22 Liu Fang et Risheng Wang sont arrivés au Québec en 1996.

23 La musicienne a notamment bénéficié d'un appui du CAC en début de carrière nord-américaine, ce qui se fait par ailleurs plus rare ces dernières années. Les raisons de cette diminution d'aide financière n'ont pas été discutées explicitement, mais Risheng Wang disait apprécier le CAC pour sa propension à chercher de nouveaux talents. Ainsi, l'on peut émettre l'hypothèse que la diminution de l'appui financier tienne en partie du passage du temps, l'effet de la «nouveauté » s'étant peu à peu estompé. 
outils de socialisation, permettant de créer des contacts, d'échanger avec d'autres collègues pour s'affirmer comme artiste, mais aussi comme individu, citoyen dans l'environnement montréalais.

Enfin, cette réflexion sur les trajectoires de musiciens migrants se conclura par des considérations linguistiques qui, même s'il s'agit principalement de musique, jouent un rôle dans cette question de positionnement dans le contexte montréalais. En effet, les compétences linguistiques interviennent dans le processus d'insertion au sein d'un nouvel environnement ${ }^{24}$. Ce sujet s'avère particulièrement sensible dans le contexte québécois où la langue française agit comme marqueur identitaire et fait l'objet d'une politique linguistique depuis 1977 afin d'affirmer et de renforcer sa place centrale dans la province (Pagé 2010, p. 4-6). La forte immigration qui transforme la réalité sociale du Québec a d'ailleurs incité une telle mesure ; en effet, l'importance des différents groupes linguistiques se trouve modifiée par les flux migratoires, et le souci de conserver un poids démolinguistique pour les francophones fait de « l'intégration linguistique des immigrants [...] un enjeu marquant dans la conjoncture linguistique actuelle au Québec » (ibid., p. 4).

Pour comprendre le rapport de chaque musicien à la langue, il suffira d'évoquer le déroulement des entretiens. Les frères Tabassian, qui ont étudié dans des institutions francophones de Montréal, maîtrisent parfaitement le français. Pour Basar, il est possible de le lire, mais elle ne le parle pas vraiment. Son niveau d'anglais a cependant permis que l'entretien prenne forme. En fait, Basar reconnaît volontiers ses lacunes, tant en français qu'en anglais, et dit avoir besoin de suivre des cours de langues ${ }^{25}$. En ce qui concerne Liu Fang, l'entrevue s'est déroulée à travers une relation triangulaire impliquant la musicienne, son mari et gérant, ainsi que la chercheuse. La présence de Risheng Wang se justifiait de par sa position dans la carrière de sa femme, mais également parce que Liu Fang ne parle pas le français, maîtrise peu l'anglais et souhaitait ainsi qu'il soit présent pour traduire certaines de ses réponses exprimées en chinois ${ }^{26}$.

Un autre participant de l'enquête a également fourni un témoignage intéressant sur cette question : il s'agit de Matthew Jennejohn, musicien originaire de Colombie-Britannique dont la langue maternelle est l'anglais. Jennejohn a participé au groupe Constantinople en arrivant à Montréal en 1998. Il ne parlait alors pas très bien le français, mais Constantinople a été selon lui un bon lieu d'apprentissage. S'il admet que cet épisode aura été très exigeant sur le plan physique - combiner

24 En ce qui concerne précisément l'insertion sur le marché du travail pour les immigrants, il appert cependant que les résultats concernant les effets de la compétence linguistique divergent selon les études (Lebeau et Renaud 2002, p. 69-70).

25 "I think my language is a handicap. I have to improve my languages, both of them [français et anglais]. I can understand French but I'm not very good. Both of them are nasty now. I have to go maybe to courses, I have to take some courses. I don't know. I don't like to speak, maybe that's why I don't speak very well. I prefer to play. This is my language » (Didem Basar, février 2012).

26 D'ailleurs, le couple a parlé de la dynamique de collaboration qui prévaut entre la musicienne et d'autres artistes, affirmant qu'avec des musiciens de haut calibre en pleine possession de leur instrument la communication verbale n'est pas nécessaire, tout se passe dans l'écoute musicale mutuelle. 
l'apprentissage sur le vif du français et la pratique musicale des journées de répétition entières lui donnait des maux de tête -, Jennejohn juge que cela aura néanmoins été bénéfique pour le reste de sa carrière et pour sa présence dans la métropole. Il a refusé d'affirmer que la connaissance du français est nécessaire pour travailler, surtout dans son principal milieu d'activité (musique ancienne) où les invités étrangers sont nombreux ${ }^{27}$, mais a néanmoins conclu en disant : « de toute façon, je pense qu'il faut apprendre le français si tu veux habiter ici » (Matthew Jennejohn, février 2012).

Les compétences linguistiques de chacun apparaissent ainsi influencées par différentes circonstances personnelles et professionnelles. Ce qui importe pour cette étude est surtout l'outil que représente la langue pour cheminer dans le milieu musical montréalais et accroître son autonomie dans le contexte québécois en général. Même si la musique est un art qui peut se passer de mots, il reste que la collaboration avec d'autres musiciens nécessite dans bien des cas une capacité à dialoguer. Par conséquent, même si les contacts se créent au fil de rencontres et d'échanges de nature musicale, la perspective linguistique n'est pas à minimiser pour autant.

En somme, ces quatre trajectoires de musiciens migrants invitent à réfléchir leur professionnalisation au Québec comme un processus multidimensionnel, où interviennent des facteurs tant musicaux qu'extramusicaux, des considérations à la fois culturelles, linguistiques, sociales, etc. Les différentes perspectives qui ont été explorées - le rapport du musicien à la tradition musicale du pays d'origine, à sa « diaspora ", au milieu montréalais à travers la question des réseaux et celle des compétences linguistiques - ne sont d'ailleurs que quelques pistes de réflexion possibles ${ }^{28}$.

\section{CONSTANTINOPLE : OUTIL D'INSERTION SOCIOPROFESSIONNELLE ET LABORATOIRE DE} DÉVELOPPEMENT D'UNE IDENTITÉ MUSICALE

Pour bien comprendre la société musicale Constantinople telle qu'elle existe aujourd'hui, il importe d'en retracer les différentes étapes de développement. Par la reconstitution du parcours chronologique de la formation, il s'agira de voir comment elle s'est construite, sur les plans structurel (administration, organisation) et musical, s'est intégrée et affirmée dans le paysage musical et culturel québécois. À travers tous les témoignages recueillis, il semble se dessiner trois phases dans l'historique $\mathrm{du}$ groupe, qui seront envisagées comme phase "Pré-Constantinople ", phase "Constantinople 1», et finalement phase "Constantinople 2 ", soit la formation à l'état actuel.

27 De plus, Montréal incarne une réalité singulière au sein du Québec. La métropole est en effet reconnue pour son bilinguisme (français et anglais) et la minorité anglophone du Québec s'y concentre (Pagé 2010, p. 4).

28 Par exemple, il serait intéressant de développer davantage la question du rapport des musiciens aux institutions québécoises (établissements d'enseignement de la musique, Conseils des arts, etc.). Il a notamment été question, avec Liu Fang, des opportunités (peu nombreuses) qu'elle avait eues ici de rencontrer des étudiants en musique dans des institutions comme les Conservatoires. 
Phase "Pré-Constantinople»

Kiya Tabassian est le directeur artistique et fondateur de Constantinople. Étudiant, il a initié ce projet avec un collègue musicien, Mike Cole. Leurs instruments, le sétar pour Kiya Tabassian et le luth renaissance pour Mike Cole, permettaient de croiser deux mondes : celui de la musique persane et celui de la musique ancienne européenne. Cette idée de « rencontre » constitue d'ailleurs une sorte de leitmotiv dans l'histoire de la formation Constantinople. D'autres étudiants se sont ensuite joints au groupe : Ziya Tabassian aux percussions, Elin Söderström à la viole de gambe. Très rapidement, c'est sous le nom Constantinople qu'ils ont proposé un programme à des maisons de la culture, ce réseau que connaissait déjà Kiya Tabassian grâce aux activités de son trio de musique persane Darvish Khan. Les projets se sont vite enchaînés, dont des enregistrements de concerts par Radio-Canada qui ont contribué à leur édifier une réputation.

Même à cette étape d'émergence, les aspirations étaient grandes pour les frères Tabassian : "Dès le début c'était à long terme [...]. Pour nous, Kiya par exemple, c'est comme une relation à vie avec Constantinople. Même à l'époque, ce n'était pas juste un groupe "Ok, on va jouer". C'était vraiment un but à long terme » (Ziya Tabassian, juin 2011). Le développement de la formation musicale n'en était par ailleurs qu'à un stade embryonnaire qui allait nécessiter de nombreux efforts pour en arriver à une véritable société musicale, qu'on peut nommer « Constantinople 1 ».

"Constantinople $1 "$

En février 2002 survient un moment décisif dans l'histoire de Constantinople : leur premier concert autoproduit au Théâtre Outremont. Kiya Tabassian confirme avoir bénéficié d'une petite subvention du CAC pour le projet, un montant qui ne couvrait cependant pas même la location de la salle. Il a toutefois tenu à aller au bout de la démarche et qualifie aujourd'hui cet épisode de " premier bon coup pour [lui] " (Kiya Tabassian, décembre 2011), puisqu'il en est sorti sans aucun déficit budgétaire ${ }^{29}$. À la suite de ce concert, l'idée de mettre sur pied des saisons s'est imposée et c'est d'ailleurs à l'automne suivant cet évènement au Théâtre Outremont que correspond le début de la première saison officielle de Constantinople (2002-2003).

Lorsque Kiya Tabassian décrit les premières années de structuration du groupe, la notion de risque est récurrente dans son discours, ce qui n'est pas sans rappeler l'un des grands axes de réflexion de Menger selon qui la carrière artistique en est une de gestion de l'incertitude, donc de gestion de risques.

Exemple audio 3 : Kiya Tabassian en entrevue. Écouter.

29 Kiya Tabassian explique que le concert a attiré un auditoire considérable et que la vente de billets a suffi à couvrir tous les frais encourus par le projet. Par ailleurs, il souligne que s'il n'a connu aucun déficit budgétaire, il n'a fait aucun profit non plus. 
D'ailleurs, " la prise de risque est une demande d'information », souligne ce même sociologue (Menger 2009, p. 17) ; le résultat agit ainsi comme une sorte de révélation de la valeur de l'artiste ou du groupe ${ }^{30}$. Par conséquent, la persévérance et l'audace de Kiya Tabassian étaient nécessaires pour découvrir si Constantinople avait véritablement une place en tant que société musicale au Québec. Qui plus est, le caractère incertain de toute initiative artistique " appartient à l'essence même des satisfactions procurées par [son] exercice » (ibid., p. 16). L'attitude de Tabassian était donc pleinement cohérente avec la nature profonde du travail créateur où «l'épreuve de l'incertitude donne [...] son épaisseur d'humanité et ses satisfactions les plus hautes » (ibid., p. 9).

Pour ses premières saisons officielles, Constantinople comptait cinq musiciens permanents : Kiya et Ziya Tabassian, Matthew Jennejohn (flûte à bec et cornetto notamment), Isabelle Marchand (succédant à Elin Söderström à la viole de gambe, mais cette dernière a regagné le groupe par la suite) et Guy Ross (oud et luth, entre autres). Le répertoire exploré était surtout constitué de musique ancienne, mais une "musique ancienne qui voyage, qui va à travers le monde, qui part de l'Espagne, mais qui va jusqu'à Samarkand, qui va jusqu'en Asie centrale... » (Kiya Tabassian, décembre 2011). Il s'agissait d'un répertoire essentiellement de tradition orale, et la vision artistique du directeur s'affirmait déjà clairement à travers les choix musicaux $^{31}$ : « quand on fait de la musique ancienne, ce n'est pas un ensemble qui se veut de reproductions historiquement correctes de la musique - parce que je n'y crois pas. C'est un ensemble qui a une approche créative » (ibid.). Ce propos a été corroboré par Jennejohn, un important témoin des premières années du groupe.

\section{Exemple audio 4: Matthew Jennejohn en entrevue. Écouter.}

Il importe de souligner que si Constantinople s'intéressait alors principalement au domaine de la musique ancienne, sa structure ne correspondait pas à ce type de formation. Le sociologue Pierre François constate que les ensembles de ce milieu musical (son étude, menée en France, peut se transposer au cas québécois) fonctionnent surtout par embauche temporaire de musiciens ${ }^{32}$, les besoins en effectif instrumental variant beaucoup d'un projet à l'autre (François 2005, p. 160). Or, en tant que formation fixe, Constantinople rappelle davantage la structure des groupes en musique populaire où la présence de chaque membre est importante (Campbell Robinson, Buck et Cuthbert 1991) ${ }^{33}$.

30 Le sociologue Nicolas-Le Strat abonde dans le même sens en affirmant que "l'artiste est confronté [au] risque permanent de la déqualification, de la non-reconnaissance » et que seule l'activité agit comme "moment de vérité de la qualité artistique » (Nicolas-Le Strat 1998, p. 112).

31 Pour un aperçu du travail de Constantinople à l'époque, voir les recensions dans La Scena musicale pour les disques Memoria Sefardi (Cardin 2002) et Terres turquoises (Deschênes 2004).

32 Dans le contexte français, il est question du marché des intermittents.

33 Par exemple, Matthew Jennejohn a affirmé que l'absence d'un des cinq musiciens fixes à l'époque pouvait compromettre la réalisation d'un projet. La participation au groupe requérait ainsi un investissement considérable de chacun en termes de temps compte tenu des nombreuses répétitions. La réalité s'avère semblable dans les groupes de musique populaire comme en attestent les résultats d'une étude transculturelle publiée dans l'ouvrage Music at the margins. Popular Music and Global Cultural Diversity (Campbell Robinson, 
En fait, Constantinople était un véritable laboratoire, un projet d'équipe dont Kiya était le principal responsable sous forme de leader, tant artistique qu'administratif. La plupart des tâches de gestion lui revenaient, mais d'autres musiciens donnaient également un coup de main ${ }^{34}$. Il semble d'ailleurs que tout le monde avait son mot à dire : " La façon dont on fonctionnait était démocratique. C'était un ensemble de musique de chambre où tout le monde avait [son] input personnel. Mais des fois, il y avait certains projets qui étaient plus l'idée de telle personne » (Matthew Jennejohn, février 2012).

$\mathrm{Au}$ total, ce sont quatre saisons complètes qui ont été tenues par la formation que nous nommons, pour le propos de cet article, "Constantinople 1 ». Leurs réseaux de diffuseurs et de partenaires (Radio-Canada, Salle Pierre-Mercure, Société du Palais Montcalm, etc.) se sont en grande partie constitués durant cette période. S'y ajoutent des contacts établis lors de tournées à l'étranger ${ }^{35}$. Construit sur un modèle peut-être mal approprié aux visées artistiques changeantes de Kiya cependant, Constantinople implosera lors de la cinquième saison et l'ensemble vivra des moments difficiles, faute d'un soutien financier adéquat.

Rupture

Lors de la saison 2006-2007, la formation perd trois de ses membres réguliers, suite à quoi des musiciens se joignent aux frères Tabassian de manière ponctuelle. Le nombre de concerts annuels passe soudainement de cinq à deux ${ }^{36}$. Interrogé sur les raisons de son départ, Jennejohn a admis que "Constantinople 1 " connaissait à ce moment beaucoup de tensions, que ce soit de nature interpersonnelle ou artistique. Les dissensions quant au choix du répertoire semblent avoir été une cause majeure de cet éclatement : à titre d'exemple, si Jennejohn était principalement intéressé par la musique ancienne européenne (plus précisément la musique baroque), il sentait que les frères Tabassian voulaient peu à peu retourner vers la musique persane ou, pour reprendre les mots de Ziya Tabassian en entretien, vers des musiques de «traditions vivantes » ${ }^{37}$.

Buck et Cuthbert 1991, p. 219). Ajoutons que la rupture de "Constantinople 1 ", qui sera traitée plus loin dans l'article, découle également de raisons évoquées dans cet ouvrage comme étant parmi les causes les plus fréquentes de dissolution d'un groupe populaire : mésentente au sein du groupe, besoin de développement personnel au-delà de ce que l'ensemble peut offrir (Campbell Robinson, Buck et Cuthbert 1991, p. 218). En somme, plusieurs éléments viennent appuyer l'hypothèse selon laquelle "Constantinople 1 » adoptait une structure (et non un répertoire) qui s'apparente entre autres au milieu de la musique populaire.

34 Kiya Tabassian a déclaré que son frère Ziya s'est occupé de la comptabilité pendant un certain temps ; Matthew Jennejohn a souligné qu'Isabelle Marchand s'est impliquée dans toutes sortes de tâches extramusicales. Aussi, le travail de booking a été principalement assumé par les membres eux-mêmes, faisant appel à leurs différents contacts. Jennejohn a évoqué quelques expériences avec des agents, mais souvent sans trop de succès, car les musiciens arrivaient à trouver plus de contrats que les agents.

35 Entre 2004 et 2006, Constantinople a notamment joué dans plusieurs provinces canadiennes, à Chypre, en Espagne, en France, en Grèce, en Italie, au Liban, au Mexique, aux Pays-Bas, en Turquie, etc. Pour plus de détails sur les tournées du groupe, voir Constantinople 2015.

36 Un concert anniversaire et un concert nommé Cri persan.

37 Kiya Tabassian n'a pas traité explicitement d'un désir de changement de répertoire, mais s'est plutôt attardé à expliquer le recentrement administratif de Constantinople à la saison 2006-2007. Par contre, son 
Pour exprimer ces divergences d'intentions musicales, Jennejohn a parlé d'une sorte de « retour aux sources » de part et d'autre.

\section{Exemple audio 5 : Matthew Jennejohn en entrevue. Écouter.}

De plus, la configuration fixe du groupe rendait obligatoire la présence de chaque musicien, ce qui a engendré des conflits d'horaire, notamment pour Jennejohn qui œuvrait aussi au sein d'autres formations de musique ancienne de tradition européenne. Ces engagements étaient importants pour des raisons financières et aussi parce qu'ils lui permettaient de conserver un contact avec le répertoire pour lequel il avait été initialement formé.

Enfin, peut-on également imputer cet éclatement à l'autorité d'un des membres, le directeur artistique en l'occurrence ? Kiya Tabassian est certes un leader né et possède une vision artistique très nette et assumée, tant dans la création que la gestion $\mathrm{du}$ groupe, mais les conclusions ne semblent pas aller en ce sens. Si Jennejohn a mentionné que de fortes personnalités se côtoyaient au sein de "Constantinople 1 ", il apparaît par ailleurs que les conflits émergeaient de toutes parts. Quant au fait qu'il ne soit resté que les frères Tabassian suite à ce moment de rupture, il ne faut pas oublier que Constantinople a toujours été l'initiative de Kiya Tabassian et qu'il en est non seulement le directeur artistique, mais aussi le principal administrateur, du moins dans les premières années.

\section{Exemple audio 6 : Kiya Tabassian en entrevue. Écouter.}

En somme, si l'autorité de Kiya est incontestable, plusieurs témoignages convergent vers l'idée d'un directeur artistique ouvert aux suggestions et à la création vécue de manière collective ${ }^{38}$.

Ce moment de rupture n'aura cependant pas marqué la fin de la société musicale. Il s'agit plutôt, outre le départ de plusieurs musiciens, d'une période d'arrêt et de réflexion pour une restructuration de ce que Kiya a nommé à plusieurs reprises "l'entreprise Constantinople». Ce moment de recentrement stratégique marque une nouvelle étape dans le processus de professionnalisation du groupe et témoigne de l'assimilation des rouages du milieu musical québécois qu'avait alors faite Kiya Tabassian. $\mathrm{Au}$ cours de cette saison 2006-2007, Constantinople a reçu une subvention spéciale du cAC de 30 000\$ provenant du Bureau de l'équité, donc notamment destinée aux

frère Ziya a davantage commenté les transformations du répertoire de la formation, parlant d'une évolution «naturelle ", car la musique persane, et plus largement les musiques de tradition orale, relèvent bien souvent de traditions très anciennes. Bref, le passage de musique ancienne à musique de traditions vivantes semble avoir été plus ou moins conscient sur le moment. C'est la manière de gérer l'ensemble afin qu'il serve bien ce type de répertoire qui relèverait davantage du choix stratégique.

38 Dans les premières saisons, certains projets étaient l'idée d'autres musiciens que Kiya Tabassian (Jennejohn a souvent fait allusion à Guy Ross à cet effet). Pour ce qui est des dernières années, Didem Basar confiait que les programmes sont en constante transformation lors des répétitions, selon les propositions des musiciens. Par exemple, la saison 2011-2012 comptait un concert en partenariat avec l'Ensemble SuperMusique - Plans d'immanence, 3 et 4 décembre 2011 - et Pierre-Yves Martel y proposait, avec d'autres instrumentistes que les frères Tabassian, des créations en musique actuelle inspirées de la musique ancienne. 
organismes issus de la "diversité culturelle ». Cet argent ne pouvait toutefois servir qu'à la gestion administrative et organisationnelle, et non à la diffusion de concerts.

C'est donc fort de cette aide financière et épaulé par un ami, Yvan St-Onge ${ }^{39}$, rencontré lors d'une formation du Commerce international des arts de la scène (CINARS) quelques années auparavant que Kiya Tabassian a pu «clarifier des choses dans [s]a tête, [...] avoir une meilleure vision de ce qu'[il] voulai[t] de Constantinople, [de s]a vie professionnelle » (Kiya Tabassian, décembre 2011). L'embauche de personnel (directeur administratif, responsable de la diffusion, attaché de presse) a permis une autonomisation de l'entreprise, de telle sorte qu'au final, cette restructuration semble en adéquation avec les intentions artistiques que Kiya entretenait au moment de la rupture ${ }^{40}$.

"Constantinople $2 "$

Dès la saison suivante (2007-2008), on assiste au retour d'une programmation d'envergure. Les projets ne sont plus aussi axés sur la musique dite ancienne et 1'on note que les collaborateurs changent fréquemment ; chaque concert fait dorénavant appel à de nouveaux musiciens, bien que certains visages réapparaissent souvent ${ }^{41}$. Kiya parle d'un " ensemble à géométrie variable " (ibid.), une formation dont, outre le noyau de base que forment les frères Tabassian, les musiciens changent au gré des projets et des besoins spécifiques à chaque programme de concert. Cela répond bien au nouveau mode de gestion choisi par Kiya, à savoir une organisation par projets, laquelle constitue un moyen de gestion du risque très présent dans les arts du spectacle (Menger 2009, p. 565-566). Cette manière de faire lui a été inspirée de sa formation chez CINARS où il a principalement côtoyé des artistes des milieux de la danse et du théâtre.

\section{Exemple audio 7 : Kiya Tabassian en entrevue. Écouter.}

Il est d'ailleurs possible de tracer quelques parallèles intéressants entre Kiya Tabassian et la figure du chorégraphe, d'une part, et celle du metteur en scène, d'autre part. Dans le milieu de la danse, le chorégraphe est celui qui constitue la troupe en choisissant ses membres. Le sociologue et danseur Pierre-Emmanuel Sorignet parle d'une méthode de sélection basée sur les affinités électives (Sorignet 2010, p. 91-92),

39 Kiya Tabassian a beaucoup insisté sur l'apport de cet homme dans sa vie de musicien et « d'entrepreneur ». Yvan St-Onge, qui avait été agent principal au CAC pendant de nombreuses années, bénéficiait d'une expérience qui a servi Kiya Tabassian dans sa façon de concevoir « Constantinople 2 ».

40 La question financière, bien qu'elle ne sera pas traitée explicitement, reste centrale à la notion de professionnalisation. Notons que Constantinople bénéficie d'un appui financier considérable en s'inscrivant désormais dans des catégories de fonctionnement pluriannuel (auprès du CAM), de fonctionnement d'organismes professionnels (CALQ), et en comptant sur l'obtention régulière de subventions du Bureau de l'équité du CAC pour les artistes issus de la "diversité culturelle ». Tout cela sans négliger un public régulier que le groupe a su bâtir depuis plus d'une dizaine d'années.

41 Françoise Atlan et Didem Basar sont deux collaboratrices plus régulières sans pour autant être membres fixes. 
c'est-à-dire l'idée de rencontres humaines qui prévaut sur la recherche de bons " techniciens ». Le chorégraphe cherche souvent des personnalités avec des univers singuliers bien avant d'embaucher des danseurs techniquement irréprochables. Kiya Tabassian tient un discours très similaire, notamment lorsqu'il parle de l'arrivée de Pierre-Yves Martel au sein du groupe ces dernières années.

\section{Exemple audio 8: Kiya Tabassian en entrevue. Écouter.}

De plus, le chorégraphe est celui qui doit constamment jongler avec les impératifs de création et les stratégies d'organisation et d'administration. Chez Kiya Tabassian, l'on sent bien cette tension entre le discours du créateur et celui de l'administrateur : "Je voulais qu'on aille dans les pays de la Méditerranée, parce que c'était pour nous - le terme "marché", je ne l'aime pas - mais c'était un marché pour nous dans le sens où la musique qu'on présentait prenait ses sources dans ces pays... » (Kiya Tabassian, décembre 2011). Le directeur artistique de Constantinople est d'abord et avant tout musicien, créateur, mais sa position au sein de sa société musicale l'oblige à raisonner en homme d'affaires et à adopter un vocabulaire conséquent : "compagnie, marché, structure, budget, fonctionnement, plan stratégique » font partie de ses préoccupations (ibid.).

Finalement, une autre réalité que Sorignet décrit dans son ouvrage est que l'autorité du chorégraphe dans la troupe passe en partie par le lien qu'il entretient avec les institutions. Cette caractéristique est également le fait de plusieurs formations du milieu de la musique. Chez Kiya, ce lien avec les institutions s'exprime à travers les demandes de subventions qu'il leur adresse, ainsi qu'à travers le rôle qu'il a joué au sein du cam pendant de nombreuses années. Son implication dans le milieu institutionnel n'est d'ailleurs pas étrangère à sa manière de gérer Constantinople, car il connaît maintenant le monde musical montréalais sous toutes ses coutures et peut ainsi positionner sa société musicale en conséquence.

\section{Exemple audio 9: Kiya Tabassian en entrevue. É Ecouter.}

En somme, le mode de sélection des collaborateurs et le rôle de Kiya au sein de l'ensemble sont des parallèles pertinents entre le milieu de la danse et Constantinople.

Quant aux rapprochements avec la figure du metteur en scène, rappelons d'abord ce que Menger dit à propos de l'homme de théâtre : "Ce[lui-ci] cumule les fonctions d'entrepreneur de projets artistiques et de créateur "(Menger 2009, p. 531) ${ }^{42}$. Le couple artiste/entrepreneur s'avère pertinent pour décrire le profil de Kiya. De plus, on retrouve au théâtre la notion d'acte collectif, de responsabilité collective chez chacun des acteurs d'une troupe : "Dans un spectacle théâtral, le rôle de chacun, la responsabilité individuelle pour la réussite collective est plus fortement perçue ; il y a plus de liens avec le responsable artistique du spectacle » (ibid., p. 520). C'est, à peu de choses près, la méthode de travail de Constantinople où les musiciens engagés pour un projet sont sollicités précisément pour leur apport sur le plan créatif, l'enri- 
chissement à travers leurs connaissances musicales respectives, leur instrument et leur univers créateur.

Ainsi, le passage de "Constantinople 1 " à sa phase actuelle est marqué non seulement par un changement dans le choix des répertoires, dans la configuration du groupe, mais aussi par une nouvelle manière de gérer administrativement la société musicale. Cette restructuration formelle tend à favoriser des objectifs de nature artistique ; elle accompagne un processus de définition d'une identité musicale bien distincte pour le groupe et pour ses musiciens.

À la lumière du parcours de Constantinople, l'on constate que pour gérer l'incertitude relative au milieu artistique, Kiya Tabassian a développé petit à petit une structure pensée précisément pour répondre à ses besoins de liberté de création. Comme il ne peut échapper aux contraintes et aux limites qu'impose le milieu artistique, Kiya Tabassian a plutôt cherché à développer une société musicale qui respecte ces balises extérieures. Le processus de structuration de Constantinople s'affiche ainsi comme une quête vers l'équilibre entre un cadre rigide de gestion, symbolisé par l'institution, et un espace de liberté de création, une sorte de "marge " artistique où les musiciens de Constantinople peuvent s'épanouir. En d'autres mots, Kiya Tabassian a su structurer sa propre liberté artistique.

Exemple audio 10 : Kiya Tabassian en entrevue. Écouter.

\section{CONCLUSION}

Cette étude aura permis de retracer le processus de professionnalisation de musiciens migrants dans le contexte montréalais, d'abord à l'échelle individuelle, puis à l'échelle collective avec Constantinople. La première partie portait davantage sur le caractère multidimensionnel du processus d'insertion du musicien migrant dans le milieu musical, alors que la seconde partie révélait comment la constitution d'un ensemble peut agir à la fois comme outil dans cette démarche de professionnalisation, mais aussi comme une sorte de laboratoire d'expérimentations, de recherche d'une identité musicale pour ses musiciens.

En effet, les différentes phases de Constantinople répondaient à de graduelles transformations dans les choix musicaux de la formation. Peu à peu, la direction artistique s'est orientée vers des "traditions vivantes ", expression qui illustre d'ailleurs bien le cheminement dont il est question, car la définition d'une identité musicale est un phénomène précisément mouvant - ou « vivant »-au cours duquel sont réappropriées les multiples sources et influences musicales qui jalonnent le parcours des musiciens. Pour ces individus migrants, plusieurs bagages culturels entrent en dialogue et font l'objet d'une réinterprétation dans une combinaison originale, comme le montre la démarche des frères Tabassian à travers Constantinople. Ces derniers puisent certes matériel et inspiration à même la musique persane, mais ils en offrent une version toute personnelle, issue notamment d'explorations avec d'autres artistes, des expériences qui ajoutent sans cesse à leur bagage de ressources de surcroît.

Ainsi, la situation du musicien migrant n'est pas le résultat d'une superposition d'apports culturels, mais constitue bien une réalité nouvelle et à part entière (Giraud 1987). Puis, les mouvements migratoires tracés par chacun s'étant révélés 
multiples et multidirectionnels - tous les musiciens migrants de cette enquête effectuent de nombreux déplacements entre pays d'origine, Canada, et parfois d'autres pays, notamment pour des tournées -, leurs parcours ne répondent ni à un schéma linéaire ni à une opposition binaire entre pays d'origine et pays d'accueil : " Les frontières n'ont plus les mêmes sens et une vision qui polarise les appartenances en termes de terre d'accueil ou terre d'origine fait peut-être fausse route » (Fortin 2000, p. 17).

Enfin, Constantinople offre un exemple d'insertion socioprofessionnelle réussie comme en témoigne la position enviable de la formation dans le paysage musical québécois aujourd'hui. Kiya Tabassian, maître d'œuvre de cette aventure, n'apparaît pas seulement comme un créateur musical, mais il s'affiche aussi comme un administrateur créatif, sa capacité à gérer et à restructurer sa société musicale au fil des réorientations artistiques en étant une preuve. L'importance de l'inventivité que souligne Menger dans la poursuite d'une telle carrière ${ }^{43}$ revient alors en force : le cheminement professionnel du musicien demande visiblement un incessant effort de création, et ce, à des niveaux que l'on ne soupçonne pas toujours si l'on s'en tient strictement à une perspective artistique.

\section{BIBLIOGRAPHIE}

Accès culture (2012), www.accesculture.com, consulté le 5 avril 2015.

Aubert, Laurent (2005), "Les passeurs de musiques. Images projetées et reconnaissance internationale », dans Laurent Aubert (dir.), Musiques migrantes, de l'exil à la consécration, Genève, Musée d'ethnographie de Genève, p. 109-129.

Campbell Robinson, Deanna, Elizabeth B. Buck, et Marlene Cuthbert (1991), Music at the Margins. Popular Music and Global Cultural Diversity, Newbury Park, Sage Publications.

Cardin, Frédéric (2002), " Memoria Sefardi. Musique d'Espagne juive et chrétienne ", La Scena musicale, vol. $7, \mathrm{n}^{\circ} 7$, www.scena.org/criticsdetails.asp?id $=775 \& \mathrm{vol}=7 \mathrm{no}=7 \&$ lan $=2$, consulté le 5 avril 2015.

Chevalier, Sophie (2007), "Destin du concept de "communauté" : de la classe sociale à la culture. Le cas de la Grande-Bretagne ", Esprit critique. Revue internationale de sociologie et de sciences sociales, vol. 10, $\mathrm{n}^{\circ}$ 1, http://espritcritique.uiz.ac.ma/Dossiers/article. asp?t03 code $=62 \&$ varticle $=$ esp 1001 article $02 \&$ vrep $=1001$, consulté le 2 avril 2015.

Clifford, James (1994), «Diasporas », Cultural Anthropology, vol. 9, nº 3, p. 302-338.

Conseil des arts de Montréal (2012), www.artsmontreal.org/fr, consulté le 5 avril 2015.

Conseil des arts du Canada (2012), http://conseildesarts.ca/, consulté le 5 avril 2015.

Conseil des arts et des lettres du Québec (2012), www.calq.gouv.qc.ca, consulté le 5 avril 2015.

Constantinople (2015), " En tournée ", http://constantinople.ca/events/categorie/en-tournee, consulté le 31 mars 2015.

Deschênes, Bruno (2004), "Constantinople Kiya Tabassian et Françoise Atlan Terres Turquoises ", La Scena musicale, vol. 9, $\mathrm{n}^{\circ}$ 7, www.scena.org/criticsdetails.asp?id=1313\&vol=9no=7\&lan=1, consulté le 5 avril 2015. 
Fortin, Sylvie (2000), "Pour en finir avec l'intégration... ", document de travail, Département d'anthropologie et Groupe recherche ethnicité et société, https://depot.erudit.org/id/000937dd, consulté le 31 mars 2015.

François, Pierre (2005), Le monde de la musique ancienne. Sociologie économique d'une innovation esthétique, Paris, Economica.

Giraud, Michel (1987), "Mythes et stratégies de la "double identité" ", L’homme et la société, no 83, p. $59-67$.

Giuriati, Giovanni, et Ki Mantle Hood (1995), «La voie du gamelan. Entretien avec Ki Mantle Hood», Cahiers de musiques traditionnelles, $\mathrm{n}^{\circ}$ 8, p. 193-214.

Heinich, Nathalie (1998), Ce que l'art fait à la sociologie, Paris, Minuit.

Hill Strategies (2005), « La diversité de la population active du secteur des arts du Canada. Une analyse des données du recensement de 2001 », Regards statistiques sur les arts, vol. 3, n 3, http://conseildesarts.ca/recherche/repertoire-des-recherches/2005/03/la-diversite-de-lapopulation-active, consulté le 5 avril 2015.

Jouvenet, Morgan (2006), «Comment "être dans les bacs". Professionnalisation et authenticité dans le monde de la musique rap ", dans Gérard Mauger (dir.), L'accès à la vie d'artiste. Sélection et consécration artistiques, Broissieux, Croquant, p. 199-236.

Lebeau, Ronald, et Jean Renaud (2002), « Nouveaux arrivants de 1989, langue et mobilité professionnelle sur le marché du travail de Montréal. Une approche longitudinale ", Cahiers québécois de démographie, vol. 31, nº 1, p. 69-94.

Le Menestrel, Sara (2012), Des vies en musique. Parcours d'artistes, mobilités, transformations, Paris, L'Hermann.

Liot, Françoise (2004), Le métier d'artiste, Paris, L'Harmattan.

Menger, Pierre-Michel (2009), Le travail créateur. S'accomplir dans l'incertain, Paris, Seuil/Gallimard.

Ministère de l'immigration et communautés culturelles du Québec (MICC) (2009), Population immigrée recensée au Québec et dans les régions en 2006. Caractéristiques générales, www.midi.gouv.qc.ca/ publications/fr/recherches-statistiques/Population-immigree-recensee-Quebec-regions-2006.pdf, consulté le 2 avril 2015.

Nicolas-Le Strat, Pascal (1998), Une sociologie du travail artistique. Artistes et créativité diffuse, Paris, L'Harmattan.

Pagé, Michel (2010), "L'intégration linguistique des immigrants au Québec », avec la collaboration de Patricia Lamarre, Étude IRPP, no 3, http://irpp.org/fr/research-studies/study-no3, consulté le 2 avril 2015 .

Perrenoud, Marc (2007), Les musicos. Enquête sur des musiciens ordinaires, Paris, La Découverte.

Radio-Canada (2010), «"Nous sommes des vôtres”. Les frères Ziya et Kiya Tabassian », www.radio-canada. ca/emissions/nous sommes des votres/2010-2011/speciale.asp, consulté le 25 mai 2011.

Ravet, Hyacinthe (2006), «L'accès des femmes aux professions artistiques. Un double droit d'entrée dans le champ musical ", dans Gérard Mauger (dir.), L'accès à la vie d'artiste. Sélection et consécration artistiques, Broissieux, Croquant, p. 151-176.

Shaker, Fouad E. (2013), "Turcs ", L'encyclopédie canadienne, www.thecanadianencyclopedia.ca/fr/ article/turcs, consulté le 31 mars 2015.

Sibaud, Laetitia (2013), Les musiciens de variété à l'épreuve de l'intermittence. Des précarités maîtrisées?, Paris, L'Harmattan.

Sorignet, Pierre-Emmanuel (2010), Danser. Enquête dans les coulisses d'une vocation. Paris, La Découverte.

Tabassian, Kiya, et Ziya Tabassian (2000), Jardin de la mémoire, disque compact, Production XXI-21 XXI-CD - 21406.

Unesco (2015), " Patrimoine culturel immatériel ", www.unesco.org/culture/ich/index. 
php?lg=fr\&pg=00003, consulté le 5 avril 2015.

Mise à jour : Octobre 2015. 